\title{
Effects of Frame Rate on Two-Dimensional Speckle Tracking Derived Measurements of Myocardial Deformation in Premature Infants
}

\author{
Aura A. Sanchez, MD ${ }^{1}$, Philip T. Levy, MD ${ }^{1}$, Timothy J. Sekarski, RDCS ${ }^{1}$, Aaron Hamvas, \\ MD ${ }^{2}$, Mark R. Holland, $\mathbf{P h D}^{3}$, and Gautam K. Singh, $\mathbf{M D}^{1}$ \\ ${ }^{1}$ Department of Pediatrics. Washington University School of Medicine. Saint Louis Children's \\ Hospital. Saint Louis, MO \\ ${ }^{2}$ Department of Pediatrics. Northwestern University Feinberg School of Medicine. Ann \& Robert \\ H. Lurie Children's Hospital of Chicago. Chicago, IL \\ ${ }^{3}$ Department of Radiology \& Imaging Sciences. Indiana University School of Medicine. \\ Indianapolis, IN
}

\begin{abstract}
BACKGROUND: Frame rate (FR) of image acquisition is an important determinant of the reliability of 2-dimensional speckle tracking echocardiography (2DSTE)-derived myocardial strain. Premature infants have relatively high heart rates (HR). The aim was to analyze the effects of varying FR on the reproducibility of 2DSTE-derived right ventricle (RV) and left ventricle (LV) longitudinal strain (LS) and strain rate (LSR) in premature infants.
\end{abstract}

METHODS: RV and LV LS and LSR were measured by 2DSTE in the apical 4-chamber view in 20 premature infants ( $26 \pm 1$ weeks) with HR $163 \pm 13 \mathrm{bpm}$. For each subject, 4 sets of cine-loops were acquired at FR of $<90,90-110,110-130$, and $>130$ frames/s. Two observers measured LS and LSR. Inter- and intra-observer reproducibility was assessed using Bland Altman analysis, coefficient of variation, and linear regression.

RESULTS: Intra-observer reproducibility for RV and LV LS was higher at FR >110 frames/s, and optimum at FR >130 frames/s. The highest inter-observer reproducibility for RV and LV LS were at FR >130 and > 110 frames/s, respectively. The highest reproducibility for RV and LV systolic and early diastolic LSR was at FR $>110$ frames/s. FR/HR ratio $>0.7$ frames/s/bpm yielded optimum reproducibility for RV and LV deformation imaging.

CONCLUSIONS: The reliability of 2DSTE-derived RV and LV deformation imaging in premature infants is affected by the FR of image acquisition. Reproducibility is most robust when

Corresponding author: Gautam K. Singh, MD One Children's Place Campus Box NWT-8116 St. Louis, MO 63110 Phone: 314-454-6095 Fax: 314-454-2561 Singh_G@Kids.wustl.edu.

Author contributions:

Concept/design (GKS, MRH, PTL, AAS), data collection (AAS, PTL, TJS), data analysis/interpretation (AAS, PTL), statistics (AAS, PTL), drafting article (AAS, PTL), critical revision and approval of the article (AAS, PTL, TJS, AH, MRH, GKS), funding secured by $\mathrm{AH}$. 
cine-loops are obtained with FR/HR ratio between $0.7-0.9$ frames/s/bpm, which likely results from optimal myocardial speckle tracking and mechanical event timing.

\section{Keywords}

Strain; Strain Rate; Frame Rate; Speckle-tracking Echocardiography; Premature infants

\section{Introduction}

The immature myocardium in premature infants is characterized by its limited systolic functional reserve and decreased compliance[1]. The assessment of ventricular function becomes challenging in this age group due to the significant changes in loading conditions that occur following birth as well as their relative fast heart rates (HR) [1, 2]. The assessment of myocardial deformation is a reliable method to study ventricular function in newborn infants and has been shown to be sensitive to the presence of early myocardial disease [3-5].

Myocardial deformation over the heart cycle can be quantified through measurements of global and segmental strains and strain rates. Strain (\%) is a unit-less measure of the fractional change in a dimension of a myocardial segment and strain rate $(\mathbf{1} / \mathbf{s})$ is the time derivative of the strain[6]. When the left ventricle (LV) contracts, the myocardium fibers shorten in the longitudinal and circumferential dimensions and thicken or lengthen in the radial direction[7]. When the right ventricle (RV) contracts, the myocardial fibers shorten predominantly in the longitudinal dimension[8].

Two-dimensional (2D) speckle tracking echocardiography (STE) enables the assessment of myocardial deformation and motion that is largely independent of geometry and angle of incidence [7, 9]. Optimum temporal resolution is an important consideration when imaging and quantifying moving structures, such as the myocardium. The frame rate (FR) used to acquire ultrasound images is the primary determinant of temporal resolution and is an important determinant of the reliability of 2DSTE-derived myocardial deformation imaging [10]. The optimal FR should be used to ensure adequate recognition of myocardial speckle pattern displacement between frames [6]. Two-dimensional STE has been shown to be feasible in premature infants, however there is a lack of standardization in the 2D image acquisition in this age group, which may affect the reliability of 2DSTE-derived myocardial deformation imaging $[3,4,11]$. The objective of this study was to determine the effects of varying acquisition FR on the reproducibility of 2DSTE-derived peak RV and LV longitudinal strain (LS), systolic strain rate (LSRs), early diastolic strain rate (LSRe), and late diastolic strain rate (LSRa) in premature infants.

\section{Methods}

\section{Study design and study population}

Twenty premature infants were prospectively enrolled from among infants participating in the Premature and Respiratory Outcomes Program, a seven-center initiative sponsored by the National Heart, Lung, and Blood Institute to identify physiologic and biochemical 
markers of adverse pulmonary outcome at 1 year of age (ClinicalTrials.gov identifier NCT01435187). All the infants that participated in the present study were enrolled in the Washington University/ Saint Louis Children's Hospital neonatal intensive care unit between September 2011 and December 2013. We obtained informed consent from parents, and the institutional review board of Washington University approved the study.

The inclusion criterion was: Premature birth (born between 23 weeks and 28 weeks 6 days gestational age). The exclusion criteria were: Congenital heart disease, except patent ductus arteriosus (PDA), hemodynamically insignificant ventricular septal defects (less than a third of the diameter of the aortic valve annulus with no evidence of left atrial or ventricular dilation due to volume overload), atrial septal defects or other congenital anomalies.

The infants included underwent echocardiograms at 32 and 36 weeks postmenstrual age (PMA) to avoid the early postnatal cardiopulmonary instability, the effect of hemodynamically significant PDA, and early mortality associated with extreme preterm birth. The demographic data collected included gestational age at birth (weeks), PMA at the time of the echocardiogram analyzed (weeks), gender, birth weight and weight at the time of the echocardiogram (kilograms). The clinical data collected included HR (beats per minute, bpm), systolic and diastolic blood pressures ( $\mathrm{mmHg}$ ), presence of PDA, respiratory support (mechanical ventilation, continuous positive airway pressure and/ or oxygen), inotropic support and administration of caffeine.

\section{Image acquisition protocol}

The image acquisition protocol for this study that is outlined below was adapted from Levy et al's. 2013 Protocol for Cardiac Strain imaging and Postprocessing Data Analysis in premature infants and from our previous experience with deformation imaging acquisition $[3,12]$. The images were acquired in decubitus position during restful period without changing the position of the infant or disturbing the hemodynamic condition to avoid/ minimize HR variation during the image acquisition (3). One designated pediatric cardiac sonographer (T.J.S.) experienced in acquiring images for 2DSTE analysis obtained all the echocardiographic images. Two-dimensional, real-time, grayscale images were obtained in all twenty infants using a commercially available ultrasound scanner (Vivid 7 and 9; General Electric Medical Systems, Milwaukee, WI, USA). The images were obtained using a transducer (7.5-12 MHz) center-frequency phased-array probe and optimized to visualize the myocardial walls. The images for the LV were acquired in a standard apical fourchamber view (Figure 1)[12]. For the RV, an apical RV-focused four-chamber view was acquired (Figure 1)[3]. In this view, the RV is centered in the imaging plane sector to define the full extent of RV free wall with no visible gaps[3].

For each subject, four sets of cine-loops were acquired at FR of $<90$ frames/sec (frames/s), 90-110 frames/s, 110-130 frames/s, and $>130$ frames/s. The maximum FR used to acquire images was 160 frames/s. As premature infants have wide variations in HR even at rest, we calculated the FR/HR ratio at the time of cine-loop acquisition (frames/s/bpm), to characterize the relationship between HR and FR pertaining to the reproducibility of 2DSTE-derived longitudinal strain. The value of this ratio was an expression of the absolute 
number of FR and HR. The units of time in these variables are different. We elected not to make a conversion to facilitate the ease of the interpretation of this ratio.

\section{Image off-line analysis protocol}

The image data were digitally stored in cine-loop format for offline analysis using vendorcustomized software EchoPAC ${ }^{\mathrm{TM}}$ (General Electric Medical Systems, Waukesha, WI, USA). Only images of excellent or good quality were analyzed, which were defined as 2D cine-loops that show the full extent of the RV and LV myocardium, with no visible drop outs, and with clear delineation of endocardium and the blood pool interface [13].

The aortic valve and the pulmonary valve ejection times measured by Doppler and were used to determine the LV and RV event timing, respectively. To ensure the efficiency of the software-generated regions of interest (ROI), we have previously identified three specific apical and basal landmarks for the LV and RV to aid the observer in the manual placement of the endocardial tracing. A "sail sign"was manually traced around the inside of the endocardial boarder[3]. For the LV, the tracing points used were (a) the septal-mitral annular hinge point, (b) the apical-septal point and (c) the lateral-mitral annular hinge point. For the $\mathrm{RV}$, the tracing points were (a) the septal-tricuspid annular hinge point, (b) the apical-septal point and (c) the lateral-tricuspid annular hinge point[3].

The ROI was optimized to cover the endocardium and the full width of the myocardium up to the epicardium and to avoid the pericardium[3]. To ensure optimal tracking the acoustic markers (speckles) had about 50 points distributed at 3-mm intervals within the ROI in myocardium[3]. The full shape of the ventricle was visualized over the entire heart cycle because the data fitting is weighted according to correlations between the original markers and the tracked markers (GE Medical Systems). To enhance the speckle-tracking capabilities, the ROI was readjusted repeatedly to avoid free wall base over excursion and tracking of the trabeculations[3].

The observer initially accepted the ROI. The EchoPAC software, based on the pulmonary and aortic valve timing, generated six specific curves that represent the measured myocardial LS and LSR of each segment in the longitudinal direction and one peak value representing the average LS and LSR from all segments (Figures 1 and 2). The integrity of myocardial speckle-tracking was visually confirmed, ascertained from the credibility of the algorithm-generated strain curve and from the tracking quality score generated by the automated tracking detection algorithm in the vendor-customized analysis software[3]. The final ROI was manually accepted and peak LS and LSR values were automatically calculated.

\section{Reproducibility analysis}

Cine-loops of the same cardiac cycles were used for inter-observer and intra-observer reproducibility analyses. Each observer performed offline analysis using the same measurement protocol[ 3,12$]$. Intra-observer variability was assessed by one investigator (A.A.S.), who repeated the analysis (blinded to the initial results) on the same cardiac cycles two weeks apart to reduce recall bias. Inter-observer variability was tested for all analyses 
by a second observer (P.T.L.) blinded to the results of the first observer. Both observers were blinded to the clinical status of the infants.

\section{Statistical analysis}

Descriptive statistics were used to present the demographic and clinical data. Categorical variables were expressed as percentages and continuous variables as means \pm standard deviation. The intra- and inter-observer reproducibility analysis for the LS and LSR measurements included: Bland-Altman plot analysis [percentage bias and 95\% limits of agreement (LOA)] and coefficient of variation $[14,15]$. The strength of agreement between the intra-observer and inter-observer measurements was evaluated using simple linear regression analysis (Pearson's correlation). $\mathrm{P}$ values $<0.05$ were considered significant. The statistical analyses were performed using SPSS version 14.0 (SPSS, Inc., Chicago, IL) MedCalc Statistics for Biomedical Research Version 12.3.0.0 (MedCalc Software, Mariakerke, Belgium).

\section{Results}

Twenty echocardiograms were analyzed in this study. A total of 320 clips obtained at different FR were analyzed. The mean gestational age of the infants at birth was 26 weeks, and the echocardiograms were obtained at a mean PMA of 35 weeks. A fourth of them had a PDA, but only in one infant, it was hemodynamically significant. Fourteen infants required respiratory support at the time of the study. None were on inotropic agents and only three were on caffeine (Table 1).

\section{Reproducibility Analysis}

The reproducibility analysis for peak LS is summarized in Table 2 and for peak LSR is summarized in Table 3. Figure 3 represents the correlation plots for the intra-observer and inter-observer relationship of RV and LV longitudinal strain.

\section{Intra-observer reproducibility}

Strain (\%):

The intra-observer analysis for the RV LS showed increased reproducibility with FR above 110 frames/s, with small coefficient of variation, percentage bias and narrow LOA. The same degree of reproducibility was seen with FR above 130 frames/s. Similarly, the LV LS reproducibility was higher for FR above 110 frames/s, without significant change when FR was increased above 130 frames/s. Cine-loops obtained with a FR/HR ratio above 0.7 frames $/ \mathrm{s} / \mathrm{bpm}$ yielded the most reproducible results of LS for both ventricles.

Strain Rate (1/s):

The intra-observer analysis for the RV LSRs, RV LSRe, and RV LSRa showed increased reproducibility with FR above 110 frames/s, with small coefficient of variation, percentage bias and narrow LOA. The same degree of reproducibility was seen with FR above 130 frames/s. The LV LSRs intra-observer reproducibility was highest with FR above 130 frames/s, but had wider LOA and higher coefficient of variation than the comparable RV 
parameters. The intra-observer reproducibility for LV LSRe and LSRa was poor. Cine-loops obtained with a FR/HR ratio above 0.7 frames/s/bpm yielded the most reproducible results of RV LSRs, RV LSRe, and RV LSRa, and above 0.9 for LV LSRs (Table 3).

\section{Inter-observer reproducibility}

Strain $(\%)$

The inter-observer analysis for the RV LS showed higher reproducibility with images acquired at FR above 110 frames/s, but optimized at FR above 130 frames/s. In comparison with the intra-observer analysis, the 95\% LOA were slightly wider, and \%bias and coefficient of variation were slightly increased. The inter-observer analysis for the LV LS showed the highest reproducibility at FR above 110 frames/s, without significant change when the FR was increased above 130 frames/s. The $95 \%$ LOA, \% bias and coefficient of variation were comparable to the values obtained with the intra-observer analyses. Cine-loop of images obtained with a FR/HR ratio above 0.7 frames/s/bpm allowed better reproducibility for RV and LV pea LS.

Strain Rate (1/s)

The inter-observer analysis for the RV LSRs, RV LSRe, and RV LSRa showed increased reproducibility with FR above 110 frames/s, with small coefficient of variation, percentage bias and narrow LOA. Comparable reproducibility was seen with FR above 130 frames/s. The LV LSRs inter-observer reproducibility was highest with FR above 130 frames/s. The LV LSRe and LSRa had poor inter-observer reproducibility. Cine-loops obtained with a FR/HR ratio above 0.7 frames/s/bpm yielded the most reproducible results of RV LSRs, RV LSRe, and RV LSRa, and above 0.9 for LV LVSRs only (Table 3).

\section{Discussion}

Our study reveals that the intra- and inter-observer reproducibility of 2DSTE-derived RV and LV LS and LSR in premature infants were (1) best when FR above 110 frames/sec were used for image acquisition; and (2) that the best test reliability was observed when the images were acquired at a FR/HR ratio between 0.7 and 0.9 frames/s/bpm.

Longitudinal strain provides a major contribution to stroke volume during systole[8]. LV and RV longitudinal deformation is extremely sensitive to the presence of early myocardial disease [5, 7]. 2DSTE-derived strain has been validated against MRI and sonomycrometry [9]. However, its reproducibility is affected by: (1) variability of image acquisition, (2) ultrasound equipment and software used for offline analysis and (3) operator post-processing analysis technique $[12,16,17]$.

In this study, we addressed the first two factors by having one experienced sonographer acquire all the images according to a defined protocol and by utilizing the same ultrasound scanner and one vendor- customized software workstation package for offline analysis. Both observers addressed the third factor by following our previously describes post-processing imaging analysis protocol $(3,12)$. The inter-subject variability was minimized by selecting a homogenous population of infants, in regards to their body size and disease-specific factors 
that may affect their ventricular function[13]. The intra-study variability in image acquisition (beat-to-beat variability) was minimized as the observers analyzed the same cardiac cycles[13]. These study design allowed us to identify the FR and HR/FR ratio that would allow the most reproducible and clinically reliable results of longitudinal strain measured by 2DSTE.

FR is considered to be one of the most important aspects of echocardiographic image acquisition[18]. STE is based on analyses of changes in speckle pattern position between frames. The FR must be high enough so that the motion of specific speckle patterns are recognizable between frames and can be followed[19]. However, if the FR is too high, then the relative motion of specific speckle patterns between consecutive frames may not be large enough to provide meaningful estimates of motion[6]. The reproducibility in older children, who have resting HRs ranging between 70 and 120 beats/min, is the most robust for images obtained with FR between 60 and 90 frames/sec [12]. Considering that premature infants usually have resting HR that normally range from 120 to 180 beats $/ \mathrm{min}$, it is imperative to discern the optimal FR of acquisition to enhance feasibility and reproducibility. Studies done in this age group have used varying FR, ranging from 50 to 120 frames/s $[3,4,11]$. This is the first study that we are aware of to assess the optimum FR needed to enhance reliability of 2DSTE derived myocardial strain in premature infants.

The average HR in our study group was around $163 \mathrm{bpm}$. The lower reproducibility at FR less than 110 frames/s found in this study, could be explained by inaccurate speckle tracking and timing of mechanical events, as with too-low FR, the speckles might move too much between frames to be recognized [19]. The highest FR used to acquire images in this study was 160 frames/s, which corresponded to a FR/HR around 0.9 frames/s/bpm. FR above 130 frames/s did not change significantly the RV strain reproducibility results, but improved the reproducibility of the LV strain parameters. This study demonstrated that LV LSRe and LSRa values were not easily reproducible in premature infants. This could be explained by the fact that some infants with elevated heart rates, the diastolic $\mathrm{E}$ and A waves generated by the software were fused, making it difficult from the user and the software algorithm to discern the exact value (Figure 2). Our lab has only been able to demonstrate high feasibility and reproducibility of systolic strain rate measurements in premature infants [3].

In addition, there might be other reasons for the effect of FR in the reproducibility of 2DSTE-derived strain parameters. The actual algorithm used by the vendor (GE EchoPac system [GE Medical Systems], Milwaukee, WI) is not known or published and it is unclear whether or not it takes account of global cardiac size or morphology in premature infants. This becomes an issue in pediatric heart disease where both size and geometry are important factors. Nevertheless, the variability in image acquisition is a major source of result discrepancy and hinders the extrapolation of research data to clinical practice.

\section{Clinical implications}

Two-dimensional STE is a non-invasive technique that has been applied to assess myocardial mechanics in children and infants with cardiac and non-cardiac disorders, [4, 19-22]. Standardization of 2D image acquisition must be clearly established before routine clinical adoption of 2DSTE imaging in premature infants. We have determined that with a 
FR/HR ratio $>0.7$, deformation imaging is a reproducible non-invasive method to assist in the characterization and monitoring of cardiac function in preterm infants. Although our study centered on infants without heart disease, our reproducibility results can be extrapolated to the assessment of ventricular function in premature infants with heart disease as we tested inter- and intra-observer variability on the same images with the only varying factor being the FR. However, more data is needed for those premature infants with single ventricle physiology.

\section{Limitations}

In this study, we exclusively analyzed the effect of FR in the reproducibility of 2DSTEderived LV and RV LS and LSR in the 4-chamber view. Our measurements did not consider the LV-anterior and posterior wall myocardial deformation (obtained in the standard 2 and 3-chamber apical views) to calculate global LV LS and LSR. Because of the geometry of the $\mathrm{RV}$, an adequate short-axis view for radial and circumferential deformation analysis is often difficult to acquire in infants. Therefore, we did not obtain such view for the LV either. Furthermore, there is a paucity of studies that used radial or circumferential strain measurements in clinical practice to measure cardiac function in infants, and those studies have not been able to demonstrate significant reliability [3, 5]. Although, radial and circumferential deformation are important components of the LV mechanics, LS is the most sensitive and reliable myocardial deformation parameter to assesse RV and LV function[3, $5,6,8]$.

This study did not compare the effect of varying FR between different vendors or vendorcustomized speckle tracking software programs on reproducibility of myocardial strain values. Recent studies demonstrated no significant variation in the measurements of longitudinal strain when using different cardiac ultrasound systems for image acquisition and analysis[23, 24]. However, none of these studies specifically measured the effect of the FR variability among the different echocardiographic equipment and proprietary software for image analysis. Additional studies are required to assess the effect of FR in the reproducibility of myocardial strain across different vendors.

Finally, 2DSTE depends on a semi-automated software program that requires operator experience for its application to research and clinical practice. There is a learning curve that is essential before introducing the technique into clinical or research studies[3].

Conclusions-The reliability of two-dimensional speckle tracking derived right and left ventricular longitudinal deformation imaging in premature infants is affected by the frame rate of image acquisition. Reproducibility is most robust when cine-loops are obtained when the frame rate/heart rate ratio is between 0.7 and 0.9 frames $/ \mathrm{s} / \mathrm{bpm}$, which likely results from optimal myocardial speckle tracking and mechanical event timing.

\section{Acknowledgments}

This study was supported by grants from the Premature and Respiratory Outcomes Program (NIH 1U01 HL1014650 and U01 HL101794). 


\section{Abbreviations}
RV
Right Ventricle
LVFW
Left Ventricle
2DSTE
Two-dimensional speckle tracking echocardiography
LS
Longitudinal strain
LSRs
longitudinal systolic strain rate
LSRe
longitudinal early diastolic strain rate
LSRa
longitudinal late diastolic strain rate

\section{References:}

1. Mertens L, Seri I, Marek J, et al. Targeted Neonatal Echocardiography in the Neonatal Intensive Care Unit: practice guidelines and recommendations for training. Writing Group of the American Society of Echocardiography (ASE) in collaboration with the European Association of Echocardiography (EAE) and the Association for European Pediatric Cardiologists (AEPC). J Am Soc Echocardiogr. 2011; 24:1057-1078. [PubMed: 21933743]

2. Rudolph, A. Congenital diseases of the heart: clinical-physiological considerations. WileyBlackwell; Chichester, United Kingdom: 2009. Perinatal and postnatal changes in the circulation; p. 25-36.

3. Levy PT, Holland MR, Sekarski TJ, et al. Feasibility and reproducibility of systolic right ventricular strain measurement by speckle-tracking echocardiography in premature infants. J Am Soc Echocardiogr. 2013; 26:1201-1213. [PubMed: 23880052]

4. El-Khuffash AF, Jain A, Dragulescu A, et al. Acute changes in myocardial systolic function in preterm infants undergoing patent ductus arteriosus ligation: a tissue Doppler and myocardial deformation study. J Am Soc Echocardiogr. 2012; 25:1058-1067. [PubMed: 22889993]

5. Nestaas E, Stoylen A, Brunvand L, et al. Longitudinal strain and strain rate by tissue Doppler are more sensitive indices than fractional shortening for assessing the reduced myocardial function in asphyxiated neonates. Cardiol Young. 2011; 21:1-7. [PubMed: 20923594]

6. Mor-Avi V, Lang RM, Badano LP, et al. Current and evolving echocardiographic techniques for the quantitative evaluation of cardiac mechanics: ASE/EAE consensus statement on methodology and indications endorsed by the Japanese Society of Echocardiography. Eur J Echocardiogr. 2011; 12:167-205. [PubMed: 21385887]

7. Geyer H, Caracciolo G, Abe H, et al. Assessment of myocardial mechanics using speckle tracking echocardiography: fundamentals and clinical applications. J Am Soc Echocardiogr. 2010; 23:351369. quiz 453-355. [PubMed: 20362924]

8. Petitjean C, Rougon N, Cluzel P. Assessment of myocardial function: a review of quantification methods and results using tagged MRI. J Cardiovasc Magn Reson. 2005; 7:501-516. [PubMed: 15881535]

9. Amundsen BH, Helle-Valle T, Edvardsen T, et al. Noninvasive myocardial strain measurement by speckle tracking echocardiography: validation against sonomicrometry and tagged magnetic resonance imaging. J Am Coll Cardiol. 2006; 47:789-793. [PubMed: 16487846]

10. Negishi K, Negishi T, Agler DA, et al. Role of temporal resolution in selection of the appropriate strain technique for evaluation of subclinical myocardial dysfunction. Echocardiography. 2012; 29:334-339. [PubMed: 22150476]

11. Elkiran O, Karakurt C, Kocak G, et al. Tissue Doppler, strain, and strain rate measurements assessed by two-dimensional speckle-tracking echocardiography in healthy newborns and infants. Cardiol Young. 2013:1-11.

12. Singh GK, Cupps B, Pasque M, et al. Accuracy and reproducibility of strain by speckle tracking in pediatric subjects with normal heart and single ventricular physiology: a two-dimensional speckle- 
tracking echocardiography and magnetic resonance imaging correlative study. J Am Soc Echocardiogr. 2010; 23:1143-1152. [PubMed: 20850945]

13. Colan SD, Shirali G, Margossian R, et al. The ventricular volume variability study of the Pediatric Heart Network: study design and impact of beat averaging and variable type on the reproducibility of echocardiographic measurements in children with chronic dilated cardiomyopathy. J Am Soc Echocardiogr. 2012; 25:842-854. e846. [PubMed: 22677278]

14. Bland JM, Altman DG. Statistical methods for assessing agreement between two methods of clinical measurement. Lancet. 1986; 1:307-310. [PubMed: 2868172]

15. Koopmans LHOD, Rosenblatt JI. Confidence intervals for the coefficient of variation for the normal and log normal distributions. Biometrika. 1964; 51:25-32.

16. Marwick TH. Will standardization make strain a standard measurement? J Am Soc Echocardiogr. 2012; 25:1204-1206. [PubMed: 23089621]

17. Koopman LP, Slorach C, Manlhiot C, et al. Assessment of myocardial deformation in children using Digital Imaging and Communications in Medicine (DICOM) data and vendor independent speckle tracking software. J Am Soc Echocardiogr. 2011; 24:37-44. [PubMed: 21095099]

18. Marwick TH. Consistency of myocardial deformation imaging between vendors. Eur J Echocardiogr. 2010; 11:414-416. [PubMed: 20164088]

19. Koestenberger M. Transthoracic echocardiography in children and young adults with congenital heart disease. ISRN Pediatr. 2012; 2012:753481. [PubMed: 22778987]

20. Di Salvo G, Rea A, Mormile A, et al. Usefulness of bidimensional deformation imaging for predicting outcome in asymptomatic patients aged $</=16$ years with isolated moderate to severe aortic regurgitation. Am J Cardiol. 2012; 110:1051-1055. [PubMed: 22728004]

21. Blanc J, Stos B, de Montalembert M, et al. Right ventricular systolic strain is altered in children with sickle cell disease. J Am Soc Echocardiogr. 2012; 25:511-517. [PubMed: 22341367]

22. Klitsie LM, Roest AA, Haak MC, et al. Longitudinal follow-up of ventricular performance in healthy neonates. Early Hum Dev. 2013; 89:993-997. [PubMed: 24080389]

23. Risum N, Ali S, Olsen NT, et al. Variability of global left ventricular deformation analysis using vendor dependent and independent two-dimensional speckle-tracking software in adults. J Am Soc Echocardiogr. 2012; 25:1195-1203. [PubMed: 22981228]

24. Manovel A, Dawson D, Smith B, et al. Assessment of left ventricular function by different speckle-tracking software. Eur J Echocardiogr. 2010; 11:417-421. [PubMed: 20190272] 

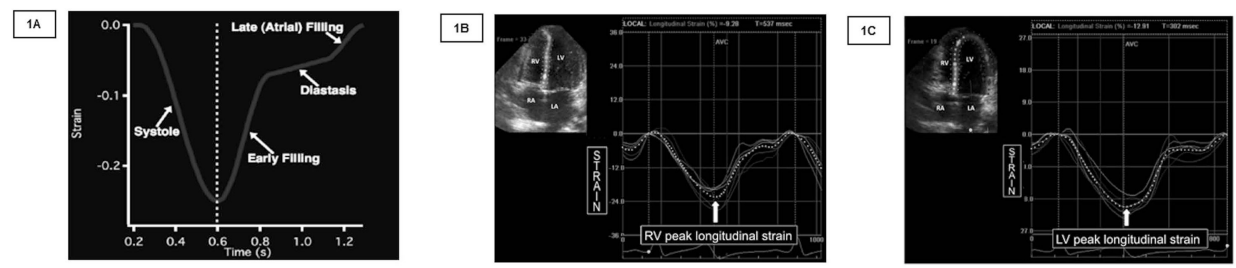

Figure 1.

(1A) The standard strain curve is depicted with the Strain (\%) on the y axis and time (s) on the $\mathrm{X}$-axis. (1B) Strain imaging of the right ventricle in a premature infant using STE. (1C) LV strain imaging graph in a premature infant using STE. Segmental strain is represented by six different curves and the average longitudinal strain by the white dotted curve. The peak of the dotted curve was considered the peak longitudinal strain value. RV: Right ventricle. LV: Left Ventricle STE: Speckle tracking echocardiography. 

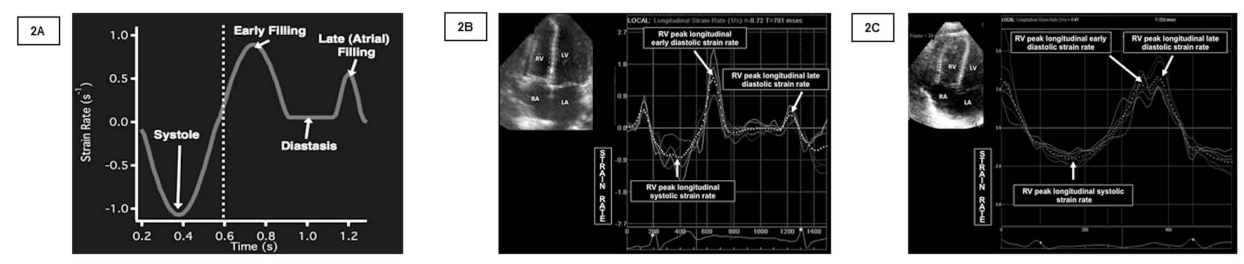

Figure 2.

(2A) The standard strain rate curve is depicted with the Strain Rate (1/s) on the $y$-axis and time (s) on the $\mathrm{x}$-axis. The longitudinal systolic strain rate, early and late diastolic strain rate are shown in the picture. (2B) Strain rate imaging of the right ventricle in a premature infant using STE with clearly defined peak global longitudinal early and late diastolic strain rate. (2C) RV strain rate imaging graph in a premature infant using STE. The peak longitudinal early and diastolic strain rate curves are fused. RV: Right ventricle. STE: Speckle tracking echocardiography. 
Interobserver Relationship
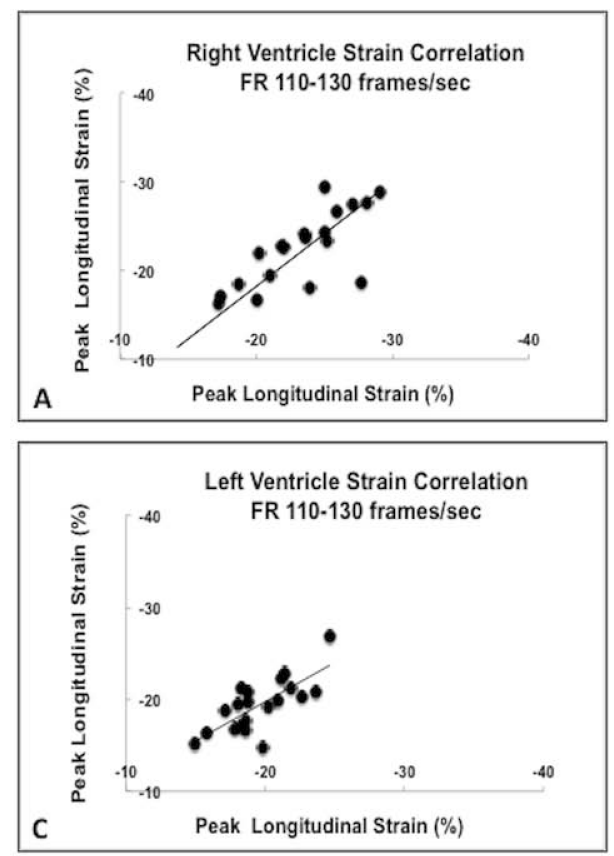

Interobserver Relationship
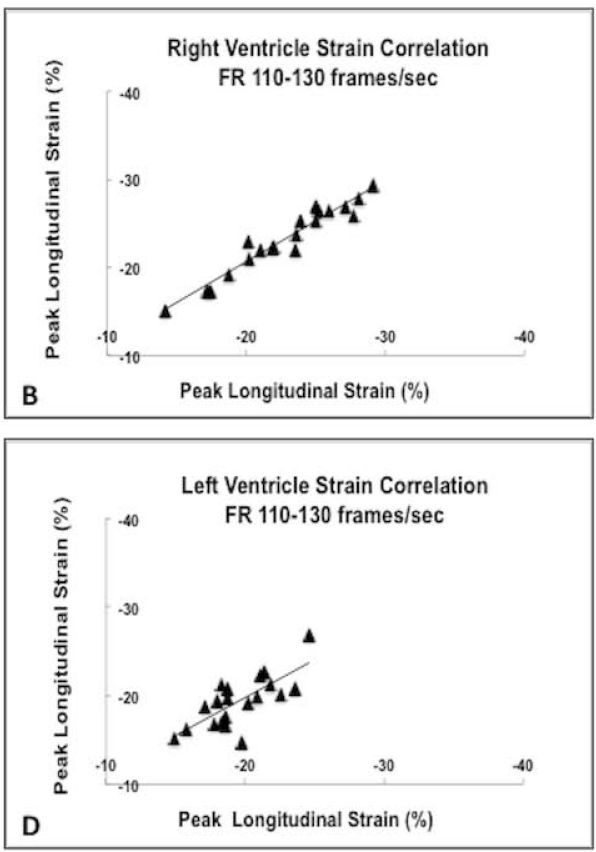

Figure 3.

(A) Correlation between the RV peak longitudinal strain values measured by the same observer (intra-observer strain correlation). (B) Correlation between the RV peak longitudinal strain values measured by two different observers (inter-observer strain correlation). (C) Correlation between the LV peak longitudinal strain values measured by the same observer (intra-observer strain correlation). (D) Correlation between the LV peak longitudinal strain values measured by two different observers (inter-observer strain correlation). RV: Right ventricle. LV: Left ventricle. 


\section{Table 1}

\begin{tabular}{|c|c|c|}
\hline \multirow{2}{*}{$\frac{\searrow}{\stackrel{D}{\vec{b}}}$} & \multicolumn{2}{|c|}{ Demographics and clinical characteristics ${ }^{l}$} \\
\hline & \multicolumn{2}{|c|}{ Table 1: Demographic and clinical characteristics } \\
\hline & Variable & $\mathbf{N}=\mathbf{2 0}$ \\
\hline & Gestational age at birth (weeks) & $26 \pm 1$ \\
\hline & Postmenstrual age at study time (weeks) & $35 \pm 3$ \\
\hline & Gender (male, \%) & $6(30)$ \\
\hline & Birth weight (Kilograms) & $0.858 \pm 0.124$ \\
\hline & Current weight (Kilograms) & $1980 \pm 0.410$ \\
\hline & Heart rate (beats per minute) & $163 \pm 13$ \\
\hline & Systolic blood pressure (mmHg) & $79 \pm 14$ \\
\hline & Diastolic blood pressure $(\mathrm{mmHg})$ & $45 \pm 13$ \\
\hline$D$ & Persistent ductus arteriosus (\%) & $5(25)$ \\
\hline & Atrial level shunt $(\%)$ & $19(95)$ \\
\hline & Respiratory support (\%) & $14(70)$ \\
\hline & Caffeine $(\%)$ & $3(15)$ \\
\hline
\end{tabular}

\footnotetext{
${ }^{1}$ Numbers are means \pm standard deviation, except for gender, persistent ductus arteriosus, atrial level shunt and respiratory support, where the number of subjects are reported with percentages within parentheses.
} 


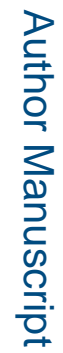

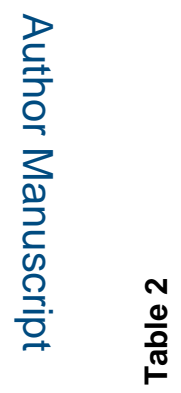

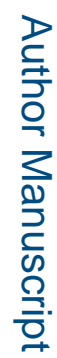

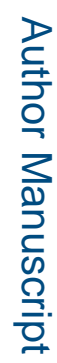

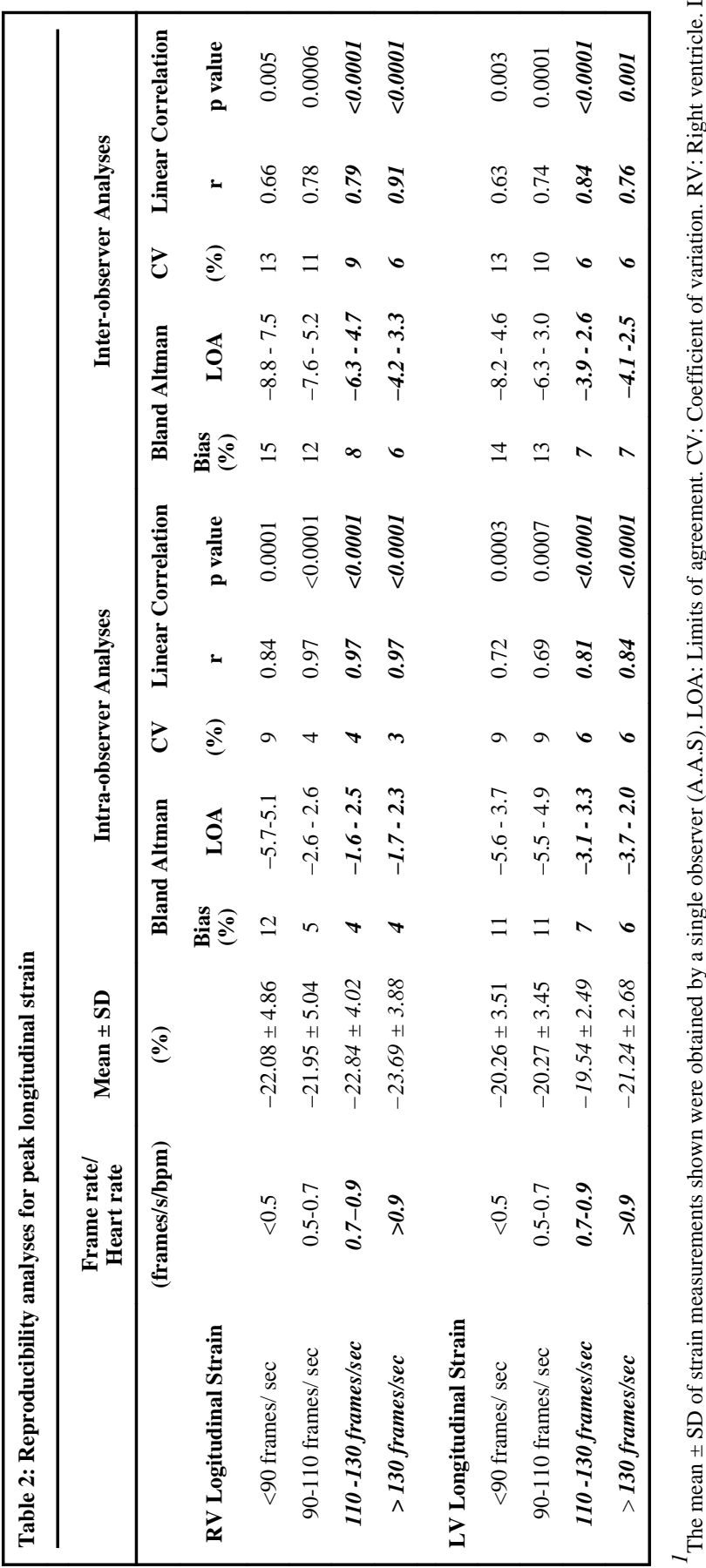

Echocardiography. Author manuscript; available in PMC 2016 May 01. 


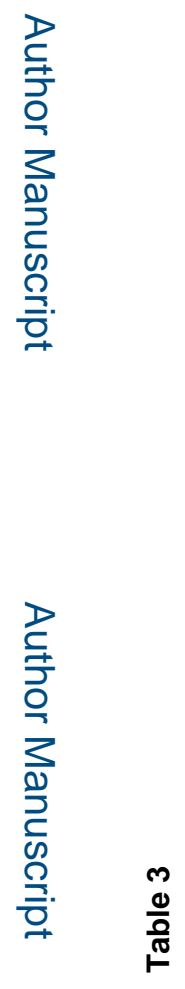

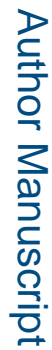

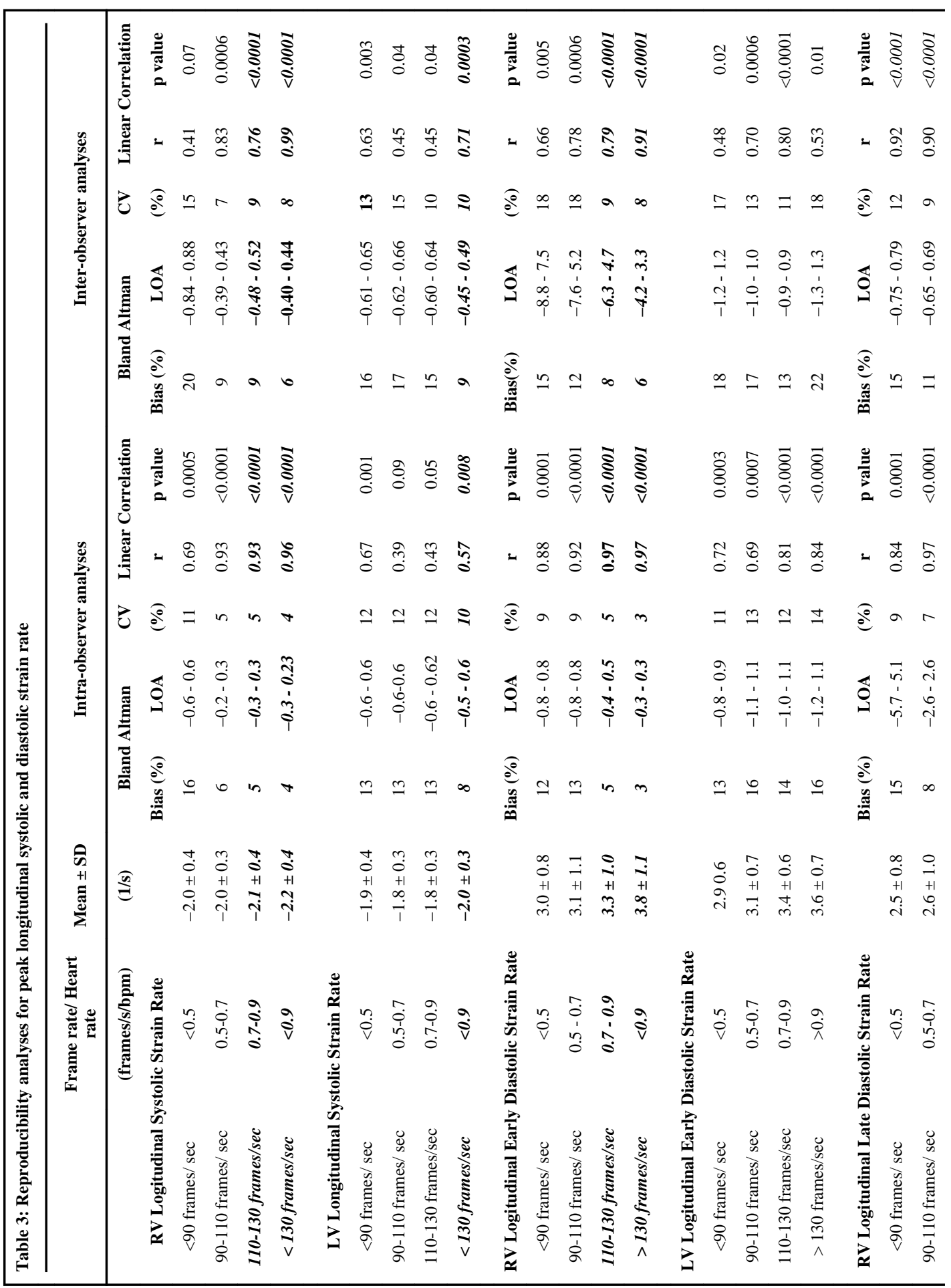

Echocardiography. Author manuscript; available in PMC 2016 May 01. 


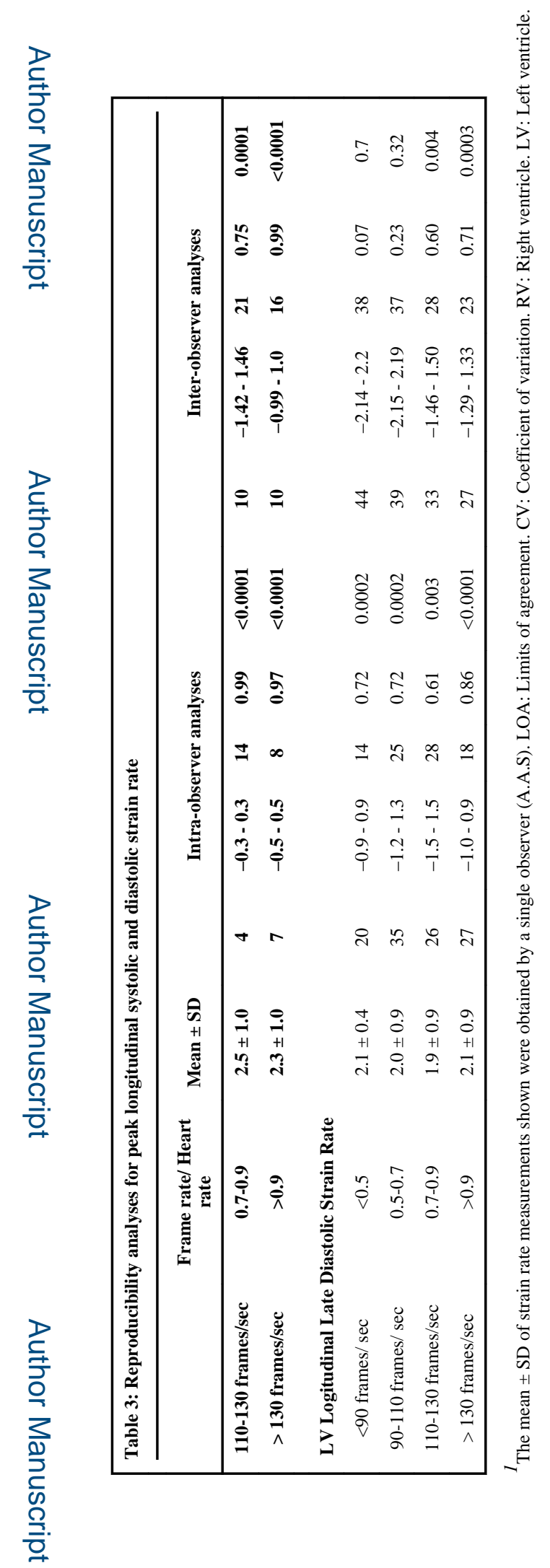

Echocardiography. Author manuscript; available in PMC 2016 May 01. 